\title{
Theoretical study of the influence of salt doping in the functioning of OLEDs
}

\author{
André Pereira, ${ }^{* a}$ Helder M. C. Barbosa, ${ }^{a}$ Helena M. G. Correia, ${ }^{a}$ Luís Marques ${ }^{a}$ and Marta \\ M. D. $\operatorname{Ramos}^{a}$
}

${ }^{a}$ Centre of Physics, Department of Physics, University of Minho,

Campus de Gualtar, 4710-057 Braga, Portugal. Fax: +351 253604 061; Tel: +351 253604330

*E-mail: andre@fisica.uminho.pt

One of the strategies to improve the efficiency of organic light emitting diodes

(OLEDs) is to dope the active organic semiconducting layer with inorganic salts, leading to the development of a hybrid organic/inorganic hetero-structure. However, it is hard to know from the experiments how each one of the electronic processes underlying the functioning of OLEDs are affected by the accumulation of inorganic ions of different sign at both organic/electrode interfaces. In order to assess these effects, we performed computer simulations by using a multi-scale model that combines quantum molecular dynamics calculations at atomistic scale with Monte Carlo calculations at mesoscopic scale. We focus our attention on the main differences obtained between doped and pristine organic layers, when bipolar charge injection occurs. Our results show a significant drop on the turn-on applied electric field while maintaining rapid response to the applied field as well as a clear increase in recombination rate and recombination efficiency far from the electrodes for the doped situation, which are responsible for the dramatic improvement of doped OLED performance found in the experiments.

\section{Introduction}

Organic light emitting diodes (OLEDs) using conjugated polymers as the active component are very attractive for their potential application in large area panel displays and general lighting. Low cost, easy fabrication and low operating voltages are some of the advantages of using these materials in the above applications. One of the parameters required to achieve high electroluminescence efficiency in organic light emitting diodes, is to have a balanced charge injection and transport throughout the active layer, which increases the probability of two charges of opposite sign recombine far from the electrodes and avoids quenching of radiative charge recombination by the electrodes ${ }^{1}$. Efficient charge injection depends on the zero-field barrier height at electrode/polymer interface (i.e. the energy mismatch between the highest occupied molecular orbital (HOMO)/lowest unoccupied molecular orbital (LUMO) of the organic and the anode/cathode Fermi Level). In general it is difficult to build OLEDs that present negligible zero-field barrier heights, due to the lack of appropriate electrodes, especially metallic cathodes.

To solve this problem, one of the strategies used is to put a sub-monolayer of a thin alkali metal halide (i.e. $\mathrm{LiF}, \mathrm{LiBr}$, $\mathrm{LiCl})^{2-4}$ between the organic layer and the metallic cathode, and to use poly(ethylenedioxy) thiophene (PEDOT) doped with poly(styrene sulphonic acid) (PSS) ${ }^{2,3}$ between the active polymer layer and the anode (usually indium tin oxide), that also works as a hole-transport layer. Although there is some controversy about the mechanism by which OLED efficiency is improved using these strategies ${ }^{5}$, several authors suggested that, for instance, LiF dissociate at the interface between the metallic cathode and poly(p-phenylene vinylene) (PPV), and lithium ions will diffuse through the polymer layer creating an hybrid ion doped region that enhances electron injection, whereas other authors suggest that the thin layer of polar metal halides between the metallic cathode creates a interfacial dipole, leading to strong band bending and thus to enhanced electron injection. Recently, another approach to increase OLED efficiency is to dope the polymer layer with small amounts of an inorganic salt, like $\mathrm{LiBr}^{6}$. The authors of this work suggest that when an external electrical field is applied, $\mathrm{Li}$ ions and $\mathrm{Br}$ ions present in the polymer film migrate to cathode and anode, respectively. The presence of a large concentration of these ions in the organic region close to the electrodes create hybrid doped charged zones that alter the electronic structure at organic/electrode interfaces, which leads to an increase in current density and light emission efficiency for significantly lower applied bias voltage as compared to pristine OLEDs. Although these experimental results clearly suggest the advantage in using inorganic salts to improve OLED performance, it is not clear how the presence of the inorganic ions affect the electronic processes of charge injection, transport and recombination, as well as, charge stored inside the active layer.

The present work aims to clarify these issues by performing computational simulations of a single-layer OLED with a doped and pristine active layer of PPV, using a multi-scale model. 


\section{Model and computer simulation details}

In our computer simulations we model the OLED functioning by considering a polymer film, with a thickness of 100 $\mathrm{nm}$, sandwiched between two perfect planar electrodes (i.e. with no impurities or defects on their surface), that were placed at $0 \mathrm{~nm}$ (cathode) and at $100 \mathrm{~nm}$ (anode), respectively. The PPV film is seen as having a spaghetti structure, where the long polymer chains consists of a large number of conjugated segments with different lengths, separated by kinks or twists ${ }^{7}$. The polymer film was built considering that the PPV segments have lengths varying from 3 to 11 monomer units, which were taken from a Gaussian distribution of lengths with a mean value of 7 monomers. Each PPV segment was randomly placed between the planar electrodes with the molecular axis parallel to the electrodes surface, as it is expected to occur for polymer films deposited by spin-coating on the electrode surface $\mathrm{e}^{7,8}$, and the minimum inter-molecular distance between neighbour segments of $0.650 \mathrm{~nm}$, based on self consistent quantum molecular dynamics calculations ${ }^{9}$. These calculations combine self-consistent quantum chemistry at Complete Neglect of Differential Overlap (CNDO) level, using a linear combination of atomic orbital and a cluster model framework, with molecular dynamics. The CNDO method, which can be viewed as a semi-empiric version of the Density Functional Theory, is embodied into the molecular dynamics method with forces calculated self-consistently at each time-step without numerical differentiation. We have chosen to use this approach because it allows one to predict charge (electron and hole) mobility along the conjugated polymer segments and molecular geometry in good agreement with the experiments and the correct trends for the variation of the ionization potential and electron affinity with the conjugation length, although their absolute values are systematic overestimated.

To study the influence of inorganic salt doping in an OLED functioning, Zhao et. al. ${ }^{6}$ used in their experiments $\mathrm{LiBr}$ with concentrations ranging from $10^{24}$ to $10^{27} \mathrm{LiBr} . \mathrm{m}^{-3}$. They observed the ionization of $\mathrm{LiBr}$ in a solution of tetrahydrofuran (THF), used to introduced this inorganic salt into the polymer film, and the formation of various amounts of ionic species dominated by lithium and bromine ions complexed with $\mathrm{LiBr}$ and THF to varying degrees. The $\mathrm{LiBr}$ in the polymer films is likely present as a mixture of ionic species with global charge neutrality and complex spatial and mass distributions, which are unknown. When the external bias voltage is applied to the electrodes, positive ions migrate towards the cathode and negative ion migrate towards the anode until the electric field caused by the ionic concentration gradient balances the applied electric field, being the lower mass ions readily transport towards the electrodes. As a result two charged layers of opposite sign are formed closed to the electrodes, separated by a region with global charge neutrality formed by the ionic screened charges in the polymer bulk. Ignoring the effect of the ionic screened charges all over the device on electron and hole injection and transport, the major effect of the inorganic salt would be ascribed to the unscreened ions placed closed to the electrodes. Since the mechanisms of ion transport in the polymer-salt systems are quite complex and cannot be handled by our model, we adopted a simple model, involving a positive charged layer close to the cathode and a negative charged layer close to the anode with a charge concentration much lower than the ensemble of the screened charges to account for the net effect of the inorganic salt doping of the polymer. Due to the lack of information in literature about the concentration and the thickness of the ion doping layer close to each one of the electrodes, we assume in this work an ion density of $6 \times 10^{24}$ ions. $\mathrm{m}^{-3}$ and a doping zone thickness of $5 \mathrm{~nm}$ near both electrodes as estimated by Jin and co-workers ${ }^{5}$ when studying the role of $\mathrm{LiF}$ in OLEDs.

To simulate bipolar charge injection into the polymer network, we consider that the injection process of electrons and holes from the opposite electrodes occurs by charge hopping from an electrode position chosen randomly to the monomer of the polymer strand which has the greatest hopping probability. In the present work, we allow for multiple charges to be injected in a sequence of events. In our model, the zero-field barrier height at polymer/electrode interface is determined by the difference between the cathode/anode work function and the average electron affinity/ionization potential of the polymer strands with different number of monomers, obtained from selfconsistent quantum molecular dynamics calculation ${ }^{10}$. The value used in this work for the zero-field barrier height for both polymer/electrode interfaces is $0.2 \mathrm{eV}$, which is of the same order of magnitude of the one estimated for similar devices used in the experiments ${ }^{11}$. Since each polymer segment has its own electron affinity and ionization potential that depend on its length ${ }^{10}$, we will have a distribution of potential barriers for both electron and hole injection. A detailed description of the effect of the of zero-field barrier height at polymer/electrode interface on the injection process can be found in Reference ${ }^{12}$.

In order to simulate the bipolar charge transport in OLEDs based on PPV, we follow the procedure described elsewhere ${ }^{8}$ and references therein. After a charge (electron or hole) be injected into a polymer strand, it moves along the strand from its injecting position until the most energetically favourable position given by the atomistic calculations, which is the centre of the strand if the local electric field (which is the sum of the applied electric field, the field created by the charge stored or in transit in the polymer network and ion distribution, and the field due to the electrode polarization) is lower than a certain value (1.53 MV.cm${ }^{-1}$ for the electron and $1.95 \mathrm{MV} . \mathrm{cm}^{-1}$ for the hole) or towards the strand-end favoured by that field otherwise. From this new position in the strand, the charge can hop by a thermally activated process to a monomer in a neighbouring polymer strand, to the injecting electrode or to the other electrode. The hopping rates associated to the electronic processes of charge injection/collection from/by the electrodes and intermolecular charge transport within the 3D-polymer network are given by: 


$$
w_{i j}=w_{o} \exp \left(\frac{R_{i j}-R_{o}}{R_{o}}\right)\left\{\begin{array} { c } 
{ \operatorname { c o s } \theta , \text { for } - \frac { \pi } { 2 } \leq \theta \leq \frac { \pi } { 2 } } \\
{ 0 , \text { for } - \frac { \pi } { 2 } > \theta > \frac { \pi } { 2 } }
\end{array} \left\{\begin{array}{c}
\exp \left(-\frac{\Delta E_{i j}}{k_{B} T}\right), \text { for } \Delta E_{i j}>0 \\
1, \text { for } \Delta E_{i j} \leq 0
\end{array}\right.\right.
$$

where the attempt-to-jump frequency, $w_{o}$, has the value $10^{9} \mathrm{~Hz}$ for intermolecular charge hopping and $10^{8} \mathrm{~Hz}$ for charge injection/collection from/by the electrodes to give the best agreement with the current density obtained in the experiments, $\theta$ is the angle between the electric force vector on the charge and the position vector along the jumping path, $R_{i j}$ is the jumping distance between the hopping sites $\mathrm{i}$ and $\mathrm{j}, R_{0}$ is the minimum intermolecular distance allowed $(0.650 \mathrm{~nm}), k_{B}$ is the Boltzmann factor, $T$ is the temperature set to $300 \mathrm{~K}$ and $\Delta E_{i j}$ is the energetic barrier that the charge carrier has to overcome during the jump. The value of $\Delta E_{i j}$ is the difference between electron affinities (for hole transport) or ionization potentials (for electron transport) of the hopping sites involved in the jumping process, since the ionization potential (electron affinity) of a negative (positive) charged polymer strand is equal to the electron affinity (ionization potential) of a neutral polymer strand, added by the effect of the local electric field on such activation energy.

The hopping probability is defined by:

$$
P_{i j}=\frac{w_{i j}}{\sum_{i \neq j} w_{i j}}
$$

and for each charge the only hopping process that takes place is the one with the greatest probability if that probability is greater than $10^{-5}$.

Recombination occurs during the simulations whenever two oppositely charge carriers meet on the same polymer strand if the local electric field does not prevent it. Because of Coulomb blockade, we assume that there is no more than one charge carrier of the same sign on each polymer strand.

The time of occurrence of the intramolecular charge transport is given by:

$$
\tau_{\text {int }}=\frac{d}{\mu E_{p}}
$$

where $d$ is the distance that the charge moves along the strand, $\mu$ is the intramolecular molibility calculated by self consistent quantum molecular dynamics and $E_{p}$ is the strength of the local electric field along the molecular axis. The values obtained for the intramolecular molibility of electrons and holes are 3.10 and $3.66 \mathrm{~cm}^{2} . \mathrm{V}^{-1} . \mathrm{s}^{-1}$, respectively. The time of occurrence of all charge hopping processes is given by:

$$
\tau_{h o p}=-\frac{\ln (x)}{w_{i j}}
$$

where $w_{i j}$ is the charge hopping frequency and $\mathrm{x}$ is a random number uniformly distributed between 0 and 1 .

A queue of increasing time steps associated to the time of occurrence of the all electronic processes in the polymer diode is used to follow the time evolution of the charges in the device. At each computer iteration the electronic process with the smallest time of occurrence takes place and is removed from the queue. This time of occurrence is then subtracted to the time of occurrence of the other electronic processes in the queue, and the time of occurrence of a new electronic process (charge injection/collection by the electrodes or charge transport) is inserted in the queue.

In the present work, all the polymer networks built have 11300 straight conjugated strands of PPV in a simulation cell with the volume of $40000 \mathrm{~nm}^{3}$. The doped polymer networks have 12 positive ions and 12 negative ions placed randomly at a distance lower than $5 \mathrm{~nm}$ from the cathode and anode, respectively, and they are not allowed to move during the simulations regardless of the local electric field. Periodic boundary conditions are applied along the directions parallel to the electrodes surface for the construction of the polymer film and the injection of charges into the polymer film. All the charges included in the simulations (electrons, holes and ions) are treated as point charges and the Coulomb interactions between them are included explicitly, as are their image interactions with the electrodes. All the results present in the next section correspond to the steady state. The results shown in the Figures were obtained after 30000 computer iterations, each one corresponding to a time step of different length according to the electronic processes involved. The simulated current, recombination and charge storage for each applied electric field is averaged over 10 different computer realizations, each one corresponding to a different polymer network to avoid an artefact of the calculations caused by any structural defect created during the building of the polymer network. We test the statistic accuracy of our result for the higher applied electric field and found no significant difference in the results obtained when the number of realizations was increased to 20. Although the number of realizations used in this work is not larger, the statistic accuracy of our results seems to be reasonable 


\section{Results and discussion}

To study the influence of doping the polymer layer with ions on OLEDs efficiency, we performed computer experiments in a pristine and doped layer of PPV, for an applied electric field ranging from $0.1 \mathrm{MV} . \mathrm{cm}^{-1}$ to 1.5 MV. $\mathrm{cm}^{-1}$. The zero-field barrier height at both polymer/electrode interfaces is chosen to be similar to avoid an unbalanced of charge injection. The results of our simulations obtained for an applied electric field of $1.5 \mathrm{MV} . \mathrm{cm}^{-1}$, at the end of approximately 1.4 microsecond of simulation time, are shown in Table 1.

Table 1: Bipolar charge injection, transport, storage and recombination inside a pristine and doped PPV layer for an applied electric field of $1.5 \mathrm{MV} . \mathrm{cm}^{-1}$ and a simulation time of approximately 1.4 microseconds.

\begin{tabular}{|c|c|c|c|c|c|c|c|}
\hline \multirow{2}{*}{$\begin{array}{c}\text { Polymer } \\
\text { layer }\end{array}$} & \multicolumn{2}{|c|}{$\begin{array}{c}\text { Effective Injected } \\
\text { Charges }\end{array}$} & \multicolumn{2}{c|}{$\begin{array}{c}\text { Charges collected by the } \\
\text { opposite electrodes }\end{array}$} & \multicolumn{2}{c|}{$\begin{array}{c}\text { Charge stored in the } \\
\text { polymer layer }\end{array}$} & \multirow{2}{*}{$\begin{array}{c}\text { Number of } \\
\text { recombinations }\end{array}$} \\
\cline { 2 - 7 } & Electrons & Holes & Electrons & Holes & Electrons & Holes & \\
\hline Doped & 185 & 194 & 15 & 23 & 26 & 27 & 144 \\
\hline Pristine & 102 & 105 & 13 & 15 & 5 & 6 & 84 \\
\hline
\end{tabular}

These results show that, for the same applied electric field and simulation time, ion doping affects mainly charge injection, storage and recombination and has little effect on charge transport towards the opposite electrode.

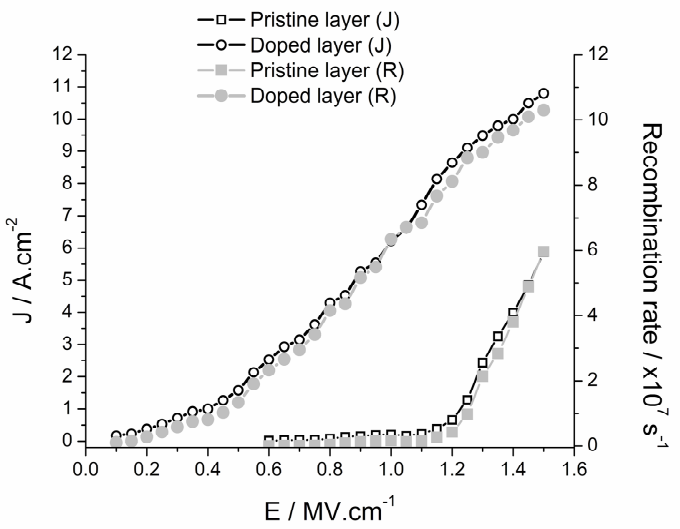

Figure 1-Dependency of current density $(\mathrm{J})$, open marks, and recombination rate (i.e. electron-hole recombination events per unit time) (R), close marks, with the applied electric field in OLEDs with a pristine (squares) and a doped (circles) polymer layer.

Fig. 1 shows the results obtained in our simulations for the dependence of the current density $(\mathrm{J})$ and recombination rate (i.e. electron-hole recombination events per unit time) (R) with the applied electric field for OLEDs with a pristine and doped polymer layer. The increase of the strength of the applied electric field increases $J$ for both polymer layers, being the same behaviour observed for $\mathrm{R}$. These results agree well with the ones obtained at experimental level for similar devices ${ }^{13}$. Our results show that ion doping strongly decreases the applied electric field necessary for charge be injected, transported and recombined in the device, which is related to the OLED turn-on voltage, as it is observed in the experiments ${ }^{6}$. Assuming that ion doping affects both radiative and non-radiative recombinations throughout the polymer layer on the same way, we found that doping the PPV layer with an inorganic salt enhances dramatically the device performance by lowering the turn-on applied electric field from the undoped value of $1.2 \mathrm{MV} . \mathrm{cm}^{-1}$ as low as $0.2 \mathrm{MV} . \mathrm{cm}^{-1}$, which corresponds to a turn-on voltage drop from 12 to $2 \mathrm{~V}$ that is significantly higher than the turn-on voltage drop from 7.4 to $3.0 \mathrm{~V}$ observed in the experiments by Zhao et. al. ${ }^{6}$ for the $\mathrm{LiBr}$ doping concentration increasing from 0 to $10^{27} \mathrm{~m}^{-3}$.

The main reason for this behaviour lies on the fact that the electric field due to the presence of ions near the electrodes reduces the potential barrier for charge injection and, therefore, charge can be easily injected into the doped polymer layer for low applied electric fields. In the case of the pristine PPV layer, the potential barrier height is high enough to limit charge injection for applied electric fields lower than $1.2 \mathrm{MV} . \mathrm{cm}^{-1}$. Although the amount of charge injected in both pristine and doped layers increases with the increase of the applied electric field, the increase of current density $(\mathrm{J})$ with the applied electric field is different for pristine and doped PPV. Our results suggest that this behaviour is due to changes in the internal electric field within the polymer layer due to ion doping, as we will discuss. 

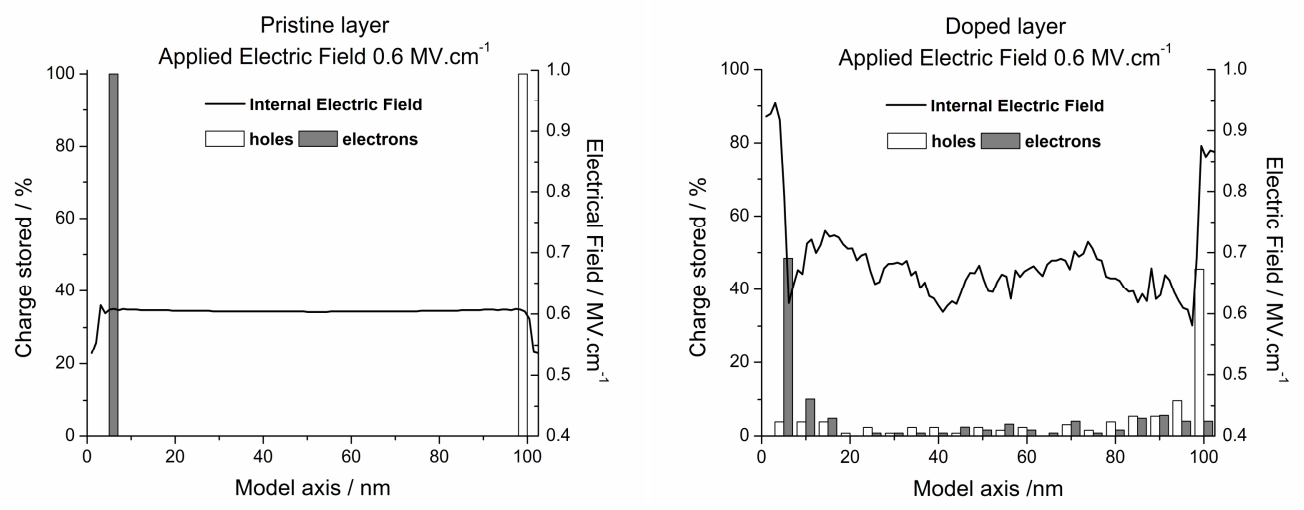

Figure 2- Fraction of holes (empty rectangles) and electrons (filled rectangles) stored and variation of the internal electric field (black line) along the direction perpendicular to the electrodes (model axis), for pristine (left hand-side) and doped (right hand-side) layers and for an applied electric field of $0.6 \mathrm{MV} . \mathrm{cm}^{-1}$.
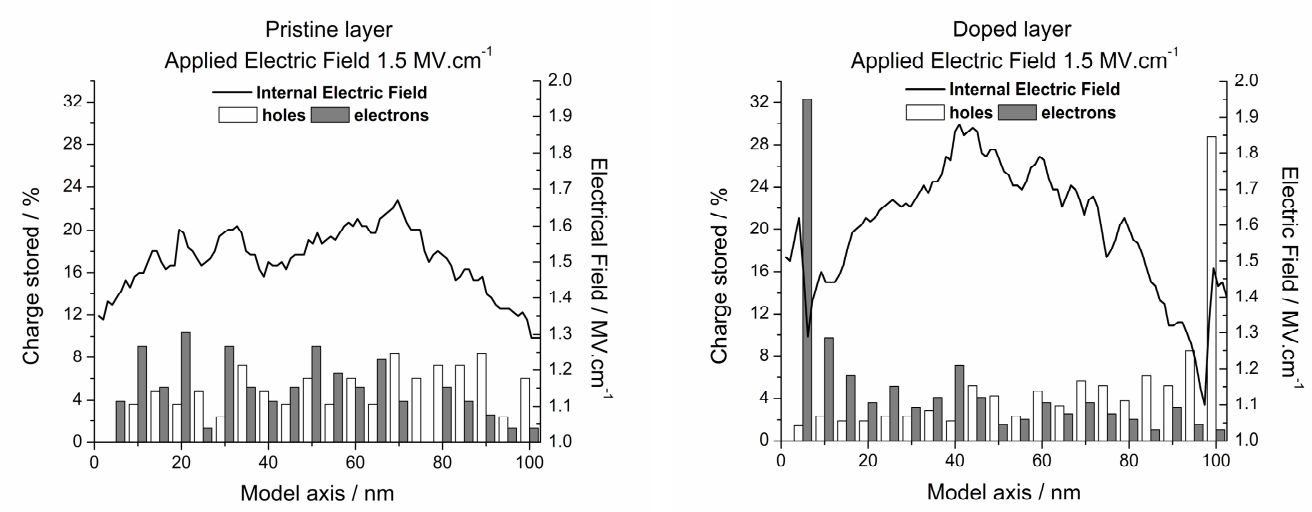

Figure 3- Fraction of holes (empty rectangles) and electrons (filled rectangles) stored and variation of the internal electric field (black line) along the direction perpendicular to the electrodes (model axis), for pristine (left hand-side) and doped (right hand-side) layers and for an applied electric field of $1.5 \mathrm{MV} . \mathrm{cm}^{-1}$.

The fact that recombination rate curve follows the current density curve, suggests that light-emitting efficiency of the device for an operating applied electric field should improve if the increase in the recombination events takes place far from the electrodes ${ }^{14-16}$, because near the electrodes there is quenching of radiative recombination. For the pristine layer and an applied electric field lower than the turn-on voltage $\left(0.6 \mathrm{MV} . \mathrm{cm}^{-1}\right)$, the injected charges are mainly stored near the injecting electrode (see Fig. 2, left-hand side) which leads to a decrease in the internal electric field on that region, being similar to the applied electrical field inside the polymer layer. As a result, this will limit the amount of charge injected and transported throughout the polymer layer, leading to a very low current density as it is shown in Fig.1. On the other hand, ion doping in the polymer layer near the electrodes leads to storage of both electrons and holes in those regions. This leads to an increase in the internal electrical field near the electrodes compared to the one observed for the pristine polymer layer (see Fig. 2, right-hand side), which will reduce the potential barrier for charge injection at polymer/electrode interfaces. Although the presence of ions in the polymer layer, leads to charge storage near them, they also assist charge transport throughout the polymer network.

They induce changes in the internal electric field, increasing its strength throughout the polymer layer that will drive charges towards the electrode opposite to injection, increasing charge transport, and thus current density, as well as the amount of charge stored within the device. 
Fig. 3 shows that, for an applied electric field higher than the turn-on voltage for both pristine and doped layers (1.5 MV. $\left.\mathrm{cm}^{-1}\right)$, there is a decrease of injected charges near the electrodes and an increase of those charges throughout the device, being this effect less pronounced for the doped layer. In this case, the internal electrical field profiles of both pristine and doped layers are similar, showing maximum values at the middle of the bulk region. The higher values reached for the doped layer, reflects both the highest amount of charge injected and stored within the polymer layer and its distribution throughout the device, compared to the pristine polymer layer. As a result there is an increase in the amount of charges of opposite sign in transit within the polymer network, which leads to an increase of the recombination rate (see Fig 1).

The noisy behaviour of the internal electric field along the direction perpendicular to the electrodes surface shown in Figures $2 b$ and 3 reflects the building up of zones of enriched and depleted discrete charge carriers incommensurate with the binning of the histograms, which would not happen if Coulomb interactions were simplified to an average space charge.

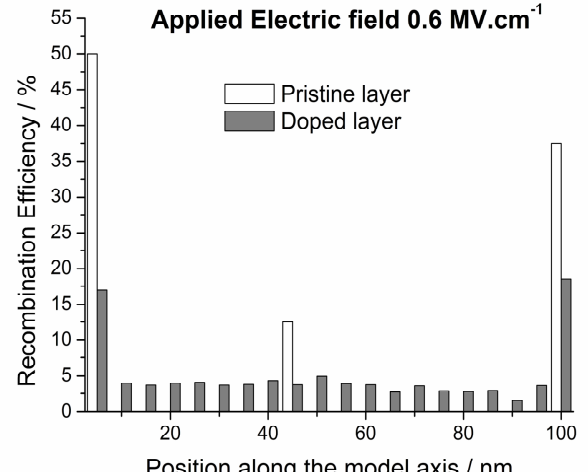

Position along the model axis / $\mathrm{nm}$

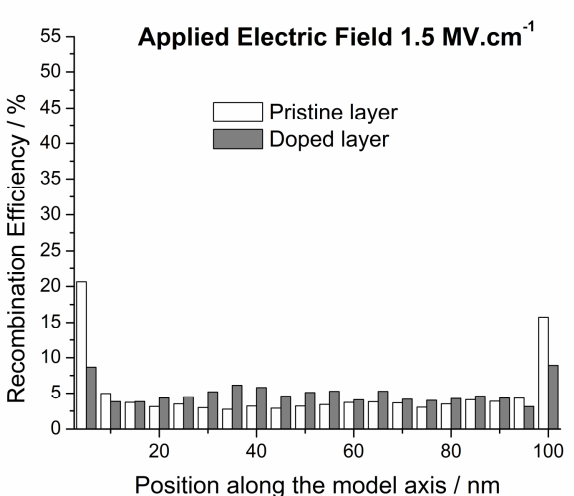

Position along the model axis / nm

Figure 4- Recombination efficiency along the axis perpendicular to the electrodes (model axis) in the pristine (empty rectangles) and doped (filled rectangles) layers, for an applied electric field of $0.6 \mathrm{MV} . \mathrm{cm}^{-1}$ (left hand-side) and 1.5 MV.cm ${ }^{-1}$ (right hand-side).

For both pristine and doped polymer layer, the increase of the applied electric field increases not only the charge recombination rate, but also leads to a more homogeneous distribution of the recombination events throughout the polymer layer (see Fig. 4). Regardless of the applied electric field, our results show that there is higher recombination efficiency near the electrodes for the pristine layer, compared to the doped one. For the doped PPV layer, charge recombination efficiency near the electrodes decreases, because charge transport towards the electrode opposite to injection becomes easier due to changes in the internal electrical field. This leads to an increase of the probability of two charges of opposite sign recombine far from the electrodes and thus increasing the probability of radiative charge recombination occurring within the polymer layer. This effect will reduce the amount of radiative charge recombination events that are quenched by the electrodes, especially near the cathode, leading to an increase of light emission efficiency ${ }^{14,15,17}$. The improvement in light emission efficiency between doped and pristine devices at the operating applied electric field can be estimated by taking into account the increase in charge recombination rate and the increase in the number of recombinations within the polymer layer excluding the ones that take place at a distance lower than $20 \mathrm{~nm}$ from the electrodes. The estimated improvement at the higher electric field of $1.5 \mathrm{MV} . \mathrm{cm}^{-1}$ is a factor of 2.5, whereas the maximum estimated improvement at the electric field threshold for light emission in the pristine device $\left(1.2 \mathrm{MV} . \mathrm{cm}^{-1}\right)$ is a factor of 25 .

Although it was expected that the presence of ions would increase the amount of recombination events at doping zone, since they would "trap" injected charges of opposite sign around them, our results show the opposite. This behaviour is justified due to a combination of different effects. Since charge injection is easier in the doped PPV layer, when new charges are injected in the polymer layer, the electric field created by them will pull out most of the trapped charges that surround the ions, increasing charge transport towards the bulk. Even though, some of the injected charges will stay "trapped" in the vicinity of the ions, eventually recombining with charges of opposite sign. The ion trapping effect is well demonstrated in Fig. 3, where is possible to see a significant difference in the fraction of holes/electrons trapped near the anode/cathode in the doped layer as compared to the pristine layer. The higher internal electric field at the middle of the doped layer and near the electrodes leads to higher charge injection and transport, which increases the recombination rate in the device. The influence of the presence of ions near the electrodes goes beyond this effect, since it increases the recombination efficiency at the middle of the polymer layer 
and reduces near the electrodes, when compared with the results obtained for the pristine polymer layer (see Fig.4), leading to an increase in light emission.

Our results explain the experimental ones obtained by Wei Zhao and J.M. White ${ }^{6}$ and show why doping the polymer layer with inorganic salts, increases current density and the light intensity, and lowers the turn-on voltage of the polymer light emitting diodes.

\section{Conclusions}

Although in our model the interaction between the ions of the doping zone and the polymer matrix has been simplified by considering that the doping ions cannot move under the action the applied electric field and they can only affect charge injection and transport through a coulomb interaction, our results show that the presence of ion doping can be responsible for the increase in OLED efficiency, especially due to changes into the internal electric field.

Our results support the idea that having high charge density near the electrodes, increases the performance of organic light emitting diodes, as previously suggested by other authors based on their experiments ${ }^{18-20}$. By doping the PPV layer with inorganic ions we have shown that it is possible to increase the amount of charges injected at low applied electric fields, by reducing the barrier height for charge injection at the polymer/electrode interface, which leads to an increase of current density and lowers the turn on voltage for OLEDs.

The change on the internal electrical field, caused by the presence of ion doping, is responsible for the increase of charge injection and transport, leading to a combined effect of increasing the recombination rate far from the electrodes and decreasing the radiative exciton quenching by the electrodes and, consequently, to an improvement in OLED efficiency.

This study also suggests new ways for the improvement of charge transport/extraction in Organic Solar Cells, namely through the introduction of ionized doping/impurities in the active layer.

\section{Acknowledgement}

This work is part of the project CONC-REEQ/443/EEI/2005 funded by the Portuguese Foundation of Science and Technology (FCT). One of us (A.P.) is also indebted to FCT for financial support under PhD. grant no. SFRH/BD/62536/2009.

\section{References}

1 R. H. Friend, R. W. Gymer, A. B. Holmes, J. H. Burroughes, R. N. Marks, C. Taliani, D. D. C. Bradley, D. A. Dos Santos, J. L. Bredas, M. Logdlund, and W. R. Salaneck, Nature, 1999, 397, 121-128.

2 W. Fahlman and W. R. Salaneck, Surface Science, 2002, 500, 904-922.

3 T. M. Brown and F. Cacialli, Journal of Polymer Science Part B-Polymer Physics, 2003, 41, 2649-2664.

4 W. J. Yoon, S. B. Orlove, R. L. Olmon, and P. R. Berger, Electrochemical and Solid State Letters, 2008, 11, J76-J78.

5 Y. D. Jin, X. B. Ding, J. Reynaert, V. I. Arkhipov, G. Borghs, P. L. Heremans, and M. Van der Auweraer, Organic Electronics, 2004, 5, 271-281.

6 W. Zhao and J. M. White, Applied Physics Letters, 2005, 87, 103503.

7 B. G. Sumpter, P. Kumar, A. Mehta, M. D. Barnes, W. A. Shelton, and R. J. Harrison, Journal of Physical Chemistry B, 2005, 109, 7671-7685.

8 H. M. C. Barbosa and M. M. D. Ramos, Materials Science and Engineering B-Advanced Functional Solid-State Materials, 2009, 165, 274-277.

9 M. M. D. Ramos, A. M. Almeida, H. M. G. Correia, R. M. Ribeiro, and A. M. Stoneham, Applied Surface Science, 2004, 238, 438-443.

10 A. M. Stoneham, M. M. D. Ramos, A. M. Almeida, H. M. G. Correia, R. M. Ribeiro, H. Ness, and A. J. Fisher, in Journal of Physics Condensed Matter; Vol. 14 (2002), p. 9877-9898.

11 V. I. Arkhipov, E. V. Emelianova, Y. H. Tak, and H. Bassler, Journal of Applied Physics, 1998, 84, 848-856.

12 H. M. C. Barbosa and M. M. D. Ramos, Thin Solid Films, 2008, 516, 1584-1587.

13 B. Geffroy, P. Le Roy, and C. Prat, Polymer International, 2006, 55, 572-582.

${ }^{14}$ H. Becker, S. E. Burns, and R. H. Friend, Physical Review B, 1997, 56, 1893.

15 J. Gruner, M. Remmers, and D. Neher, Advanced Materials, 1997, 9, 964-\&.

16 J. S. Kim, R. H. Friend, I. Grizzi, and J. H. Burroughes, Applied Physics Letters, 2005, 87, 023506.

17 A. L. Burin and M. A. Ratner, Journal of Physical Chemistry A, 2000, 104, 4704-4710.

18 W. Y. Gao and A. Kahn, Applied Physics Letters, 2001, 79, 4040-4042.

${ }^{19}$ W. Y. Gao and A. Kahn, Organic Electronics, 2002, 3, 53-63.

20 A. R. Hosseini, M. H. Wong, Y. L. Shen, and G. G. Malliaras, Journal of Applied Physics, 2005, 97, 023705. 